\title{
Bioensaio com náiades de Campylocia anceps Eaton, 1983 para avaliação dos metais cobre $(\mathrm{Cu})$ e zinco $(\mathrm{Zn})$.
}

\author{
Enide Luciana Lima Belmont ${ }^{2}$, Maria José do Nascimento Lopes ${ }^{2}$, \\ Maria do Socorro Rocha da Silva² \& Cláudio Rabelo dos Santos-Neto ${ }^{2}$
}

1. Órgão de Fomento: PPI 1-0506; PIBIC/INPA/CNPq/FAPEAM. 2. INPA - Instituto Nacional de Pesquisas da Amazônia, e-mail: belmont@inpa.gov.br (Autor correspondente), ferreira@inpa.gov.br, ssilva@inpa.gov.br, claudio@inpa.gov.br.

$$
\text { EntomoBrasilis 1(2): 33-35 (2008) }
$$

\begin{abstract}
Resumo. Em Manaus, o processo de desenvolvimento industrial e sócio-econômico, bem como a falta de programas eficientes de controle ambiental tem possibilitado a ocorrência de impactos ambientais especialmente nos igarapés urbanos. Estes impactos resultam na degradação dos recursos hídricos por meio de poluentes orgânicos e inorgânicos oriundos das atividades desenvolvidas no entorno das bacias hidrográficas. Objetivou-se então analisar os teores dos metais cobre e zinco em náiades de Campylocia anceps Eaton. O estudo foi realizado em dois afluentes na Bacia do Educandos: o primeiro, foi feita a coleta de C. anceps, está situado na Universidade Federal do Amazonas e, o segundo, foi feita a coleta da água contaminada, igarapé do Quarenta, está situado nas proximidades de fábricas do Distrito Industrial de Manaus. As náiades coletadas no igarapé universidade (água limpa) foram levadas ao laboratório de entomologia, no INPA, onde foram expostas a água contaminada do igarapé do Quarenta , nas concentrações de 100\%, 50\%,10\% e um controle com 100\% de água limpa. As C. anceps ficaram expostas a esses poluentes por seis dias. Com três e seis dias foram retiradas para a análise dos metais, no laboratório de química da água. Os teores desses nos mostraram que há uma absorção deste pelas náiades, pois quanto maior a concentração dos metais na água, maior será o valor encontrado nos organismos.
\end{abstract}

Palavras Chave: Amazonas, Euthyplociidae, igarapé do Quarenta, metal pesado.

\section{Bioassay with nymph of Campylocia anceps Eaton, 1983 for evaluation of metals copper (Cu) and zinc ( $\mathrm{Zn})$.}

Abstract. In Manaus, the process of industrial and partner-economic development, as well as the lack of efficient programs of ambient control has made possible the occurrence of ambient impacts especially in narrow river urban. These impacts result in the degradation of the hidrics resources by means of deriving organic and inorganics pollutants of the activities developed in around of the basins hidrografics. It was objectified then to analyze texts of metals couper and zinc in nymphs of Campylocia anceps Eaton. The study it was carried through in two narrow rivers in the Basin of the Educandos: the first one, was made the collection of $C$. anceps, is situated in the Federal University of Amazon and the collection of the contaminated water was made, narrow river of the Quarenta, is situated in the neighborhoods of plants of the Industrial District of Manaus. Nymphs collected in the narrow river university (clean water) had been taken to the entomology laboratory, in the INPA, where they had been displayed the water contaminated of the narrow river of the Quarenta, in the concentrations of $100 \%, 50 \%, 10 \%$ and a control with $100 \%$ of clean water. The nynfphs of $C$. anceps had been displayed to these pollutants per six days. With three and six days they had been removed for analyzes of metals, in the chemistry laboratory of the water. The texts of these in had shown them that it has an absorption of this for nymphs, therefore how much bigger the concentration of metals in the water, greater will be the value found in the organisms.

Keywords: Amazon, Euthyplociidae, havy metal, narrow river of the Quarenta.

$\mathbf{E}$ $\mathrm{m}$ Manaus, o processo de desenvolvimento industrial e sócio-econômico, bem como a falta de programas eficientes de controle ambiental tem possibilitado a ocorrência de impactos ambientais especialmente nos igarapés urbanos. Estes impactos resultam na degradação dos recursos hídricos por meio de poluentes orgânicos e inorgânicos oriundos das atividades desenvolvidas no entorno das bacias hidrográficas (Tonissi \& Espíndola 2000). Alguns destes impactos foram detectados por Couceiro et al. (2006) que avaliou os efeitos de um vazamento de óleo e despejo de esgoto doméstico no igarapé Cururu, localizado na cidade de Manaus/AM, assim, influenciando negativamente a riqueza e abundância de insetos nesses igarapés.

Nos corpos de água os poluentes inorgânicos apresentam comportamento heterogêneo podendo ser tóxicos por sua própria presença, ou pelo processo de degradação que acarretam. Isto se dá pela liberação de compostos que, assimilados pelos organismos, irão interferir nos processos fisiológicos influenciando assim nos aspectos reprodutivos, na sobrevivência e, conseqüentemente, alterando a estrutura da população/ comunidade local (Boudou \& RiBEYRE 1989; TONISSI \& ESPÍNDOLA 2000).

Os resíduos líquidos que vem sendo despejados pelo distrito Industrial de Manaus têm modificado as características dos compartimentos ambientais que fazem parte da região do igarapé do Quarenta, aonde o Zn, Pb e Ni chegaram a apresentar valores até 150 vezes maiores do que os níveis recomendados pelo CONAMA, e a concentração de metais pesados nos solos (o-8o $\mathrm{cm}$ ) está muito acima dos solos em condições naturais (SAMPAIO et al. 2000).

Em geral, o cobre, é pouco absorvido pela maioria das espécies, a retenção deste elemento depende de sua forma química e dos níveis de outras substancias minerais e orgânicas. O cobre ocorre em águas potáveis na foram do íon cuprico. De acordo com Siqueira \& Braga (2000), o zinco é um metal encontrado na natureza como sulfeto, podendo também estar associado a outros metais principalmente chumbo $(\mathrm{Pb})$, cádmio $(\mathrm{Cd})$, cobre $(\mathrm{Cu})$ e ferro $(\mathrm{Fe})$. A bioacumulação de zinco em organismos aquáticos depende dos níveis tróficos que estes ocupam na cadeia alimentar aquática, sendo que, a bioacumulação é maior nos organismos bentônicos que em organismos pelágicos (EYsink et al. 1988; Siqueira \& Braga 2000).

Os organismos aquáticos estão representados por micro e macroinvertebrados como os anelídeos, moluscos e artrópodes, que tem como representantes aquáticos os insetos. Dentre os insetos aquáticos, a ordem Ephemeroptera compreende organismos que vivem associados a rochas, troncos ou na vegetação submersa. São detritívoros e raspadores, alimentandose de matéria orgânica fina, sendo de muita importância, pois servem de alimento para outros macroinvertebrados e são bioindicadores da qualidade da água (MCCAFFERTY 1981).

Bioensaios de toxicidade são utilizados para avaliar os efeitos tóxicos potenciais de contaminantes sobre os organismos vivos. Estes efeitos podem ser avaliados através de respostas letais (mortalidade) ou subletais (fertilização, crescimento e comportamento). As espécies mais utilizadas nestes ensaios são os peixes de água doce (Cheirodon pisciculus Girard, 1855), crustáceos de água doce (Daphnia magna Straus, 1820, Daphnia pulex, Goktepe e Plhak, 2002), crustáceos marinhos (Ampelisca araucana Gallardo 1962, Emerita analoga Stimpson 1857), moluscos bivalves marinhos (Aulacomya ater Molina, 1782), Ouriços do mar (Arbacia spatuligera Valenciennes, 1846), larvas 
de Diptera (Chironomidae), algas e zooplâncton, não havendo no Brasil, estudos tratando especificamente de bioacumulação em Ephemeroptera (INSPEC 2007).

Estudos realizados na América do Norte, com bioacumulação em Ephemeroptera foi feito por Michaud et al. (2005), que trabalhou com Hexagenia limbata (Ephemeridae), no Canadá e Beretov \& Liess (2004), que estudaram Coeon dipterum (Baetidae) na Rússia.

Os teores de metais pesados verificados nos corpos de água da bacia do Educandos associado aos prejuízos que estes elementos podem acarretar aos organismos bentônicos, ao ambiente e à população humana, por si só justificam a realização de programas de biomonitoramento. Neste contexto, este estudo é pioneiro no Brasil, e, fornecerá subsídios para programas de controle ambiental buscando evitar a degradação dos recursos hídricos. Neste contexto, o presente estudo teve como objetivo avaliar a acumulação dos metais cobre e zinco em náiades de Campylocia anceps.

\section{Material e Métodos}

Área de estudo. O estudo foi realizado em um igarapé localizado na Universidade Federal do Amazonas (UFAM) (S 30 05' 55.12" e W 59058 ' 11.78"), e igarapé do Quarenta localizado no Distrito Industrial, próximo ao conjunto Nova República (S o3o 06' 2,6" e W 590 57' 17,6”). Os dois afluentes estão localizados na Bacia do Educandos, que é constituída por 33 igarapés e possui uma extensão total de 48,54 km.

As náiades de Campylocia anceps Eaton, 1983 utilizadas no experimento foram coletadas em um igarapé natural da Universidade Federal do Amazonas, que é um igarapé de águas pretas com características de ambiente não poluído, apresentando vegetação marginal e folhiço no fundo de seu leito.

O sedimento e água foram coletados em um igarapé da bacia hidrográfica do Educandos, nas proximidades da indústria Multiplast, no bairro Nova República. Suas águas contêm resíduos industriais e matéria orgânica provenientes das fábricas do Distrito Industrial de Manaus e dos conjuntos habitacionais localizados a montante.

Metodologia para coleta e criação das náiades de C. anceps. Náiades de $C$. anceps (Euthyplociidae) foram capturadas com auxílio de uma rede entomológica aquática em forma de D (rapiché). O folhiço foi coletado com o rapiché e lavado abundantemente para retirar o excesso de sedimento fino. As folhas foram passadas para um balde de 20L, transferidas em pequenas porções para bacias brancas e efetuada uma triagem inicial. Nesta triagem retiravam-se os predadores (Odonatas, Megaloptera, Blattaria, Plecoptera, etc.), para evitar a perda dos Ephemeroptera. Os Euthyplociidae encontrados foram separados, sendo colocados em um pote de isopor, com a água do igarapé e folhas para que eles não ficassem estressados. O restante do material foi levado para o laboratório nos próprios baldes, para complementar a triagem dos Euthyplociidae.

Após a coleta, as náiades foram levadas para a casa de criação de insetos aquáticos, situada em área do campus II do INPA. Este é um local aberto, com bastante ventilação, para evitar o desenvolvimento de fungos nas náiades.

Estas foram colocadas em frascos confeccionados com garrafas plásticas recicláveis, tipo "pet”, contendo 50mL de água. Cada frasco continha determinadas concentrações:

a) água limpa (controle) do igarapé natural;

b) 10\% de água de igarapé contaminado;

c) $50 \%$ de água de igarapé contaminado;

d) $100 \%$ de água de igarapé contaminado.

Utilizou-se 10 náiades para cada concentração do experimento e controle. Os experimentos foram realizados tomando por base Michaud et al. (2005).
Metodologia para a digestão das naiades de $C$. anceps. No laboratório de química, as naiades de C. anceps foram colocadas em cadinhos de porcelana lavados (água corrente, HNO a $10 \%$ e água destilada), calcinados (em mufla à $400^{\circ}$ ) e, pesados em uma balança analítica. Em seguida as naiades foram pesadas (peso úmido) e colocadas em uma estufa, a $70^{\circ} \mathrm{C}$ por 24 horas para a obtenção do peso constante. Após a secagem foi realizada a digestão do material. Para a digestão as amostras foram maceradas com um pilão de porcelana, colocadas em Beckers contendo $40 \mathrm{~mL}$ de água destilada e $5 \mathrm{~mL}$ de Peridrol de Hidrogênio. Então, foram levadas à chapa aquecedora para a completa digestão do material. Este processo é bastante lento, levando de um a dois dias para que o material seja totalmente digerido. $\mathrm{O}$ material foi filtrado através de um funil de vidro com um papel filtro e colocado em balão de fundo chato, em seguida completado com água destilada ate $50 \mathrm{~mL}$, então foram feitos leves movimentos para homogeneizar e transferidos para pequenos potes com tampa.

Em seguida, foram levados para o laboratório de química da água para a análise dos metais. Foi utilizado o Espectrofotômetro de Absorção Atômica para analisar os teores dos metais $\mathrm{Cu}$ e Zn.

\section{Resultados e Discussão}

Teor dos metais nos igarapés em estudo. No igarapé natural (UFAM) o valor de $\mathrm{Cu}$ foi de $0,9 \mathrm{mg} / \mathrm{L}$ e no igarapé do Quarenta que foi de $1,1 \mathrm{mg} / \mathrm{L}$. O valor de $\mathrm{Zn}$ no igarapé natural (UFAM) foi de 1,3 mg/L e, no igarapé Quarenta (poluído) foi de $2,5 \mathrm{mg} / \mathrm{L}$ (Tabela 1 ).

Verificamos assimqueosvalores, noigarapédoQuarenta, de $\mathrm{Cu}$ e $\mathrm{Zn}$ são baixos comparados com os resultados de Silva (1999) que foram de $3,79 \mathrm{mg} / \mathrm{g}$ e 33,0 mg/g, respectivamente.

Tabela 1. Teores de Na e K (mg/L) no igarapé da UFAM e no igarapé do Quarenta.

\begin{tabular}{lcc}
\hline & $\mathbf{C u}(\mathbf{m g} / \mathbf{L})$ & $\mathbf{Z n}(\mathbf{m g} / \mathbf{L})$ \\
\hline Igarapé natural (UFAM) & 0,9 & 1,3 \\
Igarapé poluído (Quarenta) & 1,1 & 2,5 \\
\hline
\end{tabular}

\section{Teores dos metais nas náiades de C. anceps}

Zinco. Em C. anceps, o zinco mostrou-se crescente de acordo com o aumento da concentração da água poluída. As náiades do controle apresentaram uma média do teor em $0,030 \mu \mathrm{g} / \mathrm{g}$, em $10 \%$ de concentração da água poluída este valor diminuiu cerca de $0,008 \mu \mathrm{g} / \mathrm{g}$ (valor $0,016 \mu \mathrm{g} / \mathrm{g}$ ), em $50 \%$ de concentração a média foi de $0,061 \mu \mathrm{g} / \mathrm{g}$ e, em 100\% a média foi de $0,097 \mu \mathrm{g} / \mathrm{g}$ (Tabela 2). Miesbauer (2001), trabalhando com Baetidae em um rio contaminado da Áustria, observou que as concentrações de $\mathrm{Zn}$ estavam significativamente altas $(60 \mathrm{mg} / \mathrm{kg}=60.000 \mu \mathrm{g} /$ g), comparadas com o trecho limpo do rio. Juntamente com o presente trabalho, observou-se que há uma alta concentração de $\mathrm{Zn}$, porém não são tão altos quanto os valores verificados por Miesbauer. A acumulação de metal deve-se, provavelmente, pelo comportamento alimentar específico de cada espécie, portanto há a entrada de diferentes metais na dieta do organismo.

Tabela 2. Teores $(\mu \mathrm{g} / \mathrm{g})$ de $\mathrm{Zn}$ assimilados pelas náiades de $C$. anceps expostas à água poluída.

\begin{tabular}{ccccc}
\hline \multirow{2}{*}{ Náiades } & \multicolumn{4}{c}{ Concentrações } \\
\cline { 2 - 5 } & $\mathbf{1 0 0 \%}$ & $\mathbf{5 0 \%}$ & $\mathbf{1 0 \%}$ & Controle \\
\hline 1 & 0,007 & 0,007 & 0,01 & 0,004 \\
2 & 0,009 & 0,009 & 0,01 & 0,005 \\
3 & 0,02 & 0,022 & 0,01 & 0,008 \\
4 & 0,03 & 0,03 & 0,01 & 0,02 \\
\hline
\end{tabular}

continua... 


\begin{tabular}{ccccc}
\hline \multirow{2}{*}{ Náiades } & \multicolumn{4}{c}{ Concentrações } \\
\cline { 2 - 5 } & $\mathbf{1 0 0 \%}$ & $\mathbf{5 0 \%}$ & $\mathbf{1 0 \%}$ & Controle \\
\hline 5 & 0,03 & 0,03 & 0,02 & 0,02 \\
6 & 0,05 & 0,05 & 0,02 & 0,03 \\
7 & 0,06 & 0,05 & 0,02 & 0,04 \\
8 & 0,07 & 0,07 & 0,02 & 0,05 \\
9 & 0,2 & 0,15 & 0,02 & 0,06 \\
10 & 0,5 & 0,2 & 0,02 & 0,07 \\
\hline Média & $\mathbf{0 , 0 9 7 9}$ & $\mathbf{0 , 0 6 1 8}$ & $\mathbf{0 , 0 1 6}$ & $\mathbf{0 , 0 3 0 7}$ \\
\hline
\end{tabular}

Média do CONAMA no 020/86 para Zn: 0,18 mg/L

Cobre. Quanto aos teores de cobre $(\mathrm{Cu})$ nas náiades de C. anceps, se observa que na concentração de 10\% de água poluídas, os teores foram menores do que no controle, $0,01 \mu \mathrm{g} / \mathrm{g}$. A concentração de 50\% de água poluída mostrou valores elevados, obtendo uma média de 0,026 $\mu \mathrm{g} / \mathrm{g}$. Em 100\% de água poluída, os teores foram de $0,036 \mu \mathrm{g} / \mathrm{g}$. Não são valores tão altos quando comparados com Miesbauer (2001), que verificou teores de $\mathrm{Cu}$ em náiades de Heptageniidae, que obtiveram teores de $110 \mathrm{mg} / \mathrm{Kg}$.

AraUCo et al. (2005), avaliaram a toxicidade de sulfato de cobre em Daphnia, observando que esta, apresenta uma média de absorção de $0,257 \mathrm{mg} / \mathrm{L}$ de sulfato de cobre.

Tabela 3. Teores $(\mu \mathrm{g} / \mathrm{g})$ de $\mathrm{Cu}$ assimilados pelas náiades de C. anceps expostas à água poluída.

\begin{tabular}{ccccc}
\hline \multirow{2}{*}{ Náiades } & \multicolumn{4}{c}{ Concentrações } \\
\cline { 2 - 5 } & $\mathbf{1 0 0 \%}$ & $\mathbf{5 0 \%}$ & $\mathbf{1 0 \%}$ & Controle \\
\hline 1 & 0,008 & 0,001 & 0,01 & 0,004 \\
2 & 0,009 & 0,007 & 0,01 & 0,005 \\
\hline 3 & 0,01 & 0,019 & 0,01 & 0,008 \\
8 & 0,02 & 0,021 & 0,01 & 0,02 \\
\hline 4 & 0,03 & 0,03 & 0,01 & 0,02 \\
7 & 0,09 & 0,02 & 0,01 & 0,03 \\
6 & 0,06 & 0,03 & 0,01 & 0,04 \\
\hline 9 & 0,06 & 0,04 & 0,01 & 0,05 \\
\hline 5 & 0,04 & 0,048 & 0,01 & 0,06 \\
\hline 10 & 0,04 & 0,05 & 0,01 & 0,07 \\
\hline Média & $\mathbf{0 , 0 3 6 2}$ & $\mathbf{0 , 0 2 6 6}$ & $\mathbf{0 , 0 1}$ & $\mathbf{0 , 0 3 0 7}$ \\
\hline Ḿdia do
\end{tabular}

Média do CONAMA ${ }^{\circ}$ 020/86 para Cu: 0,02 mg/L

Análise de Correlação de Person. A correlação de Pearson entre a massa/Zn mostrou que há correlação entre as variáveis (controle, 10\%, 50\%, 100\% de água poluída) e a massa dos organismos (Tabela 4 ).

Tabela 4. Correlação de Pearson entre massa do organismo e concentrações utilizadas no experimento.

\begin{tabular}{ccc}
\hline Concentrações & $\begin{array}{c}\text { Correlação de } \\
\text { Pearson }\end{array}$ & Tipo de correlação \\
\hline Controle & 0,8020 & Forte e positiva \\
$50 \%$ & $-0,173$ & Fraca e negativa \\
$100 \%$ & 0,8975 & Forte e positiva \\
\hline
\end{tabular}

As náiades de $C$. anceps se constituem em uma importante ferramenta para estudos de bioacumulação nos igarapés de Manaus devido seu tamanho e abundância. No entanto os experimentos devem ser realizados nos períodos mais seco do ano que corresponde ao período no qual são encontradas com maior abundância.

Estes organismos reagiram acumulando os metais tóxicos $(\mathrm{Zn}$ e $\mathrm{Cu})$ verificando-se que quanto maior o período de exposição do organismo ao poluente maior a taxa de absorção.

Arauco, L.R.R., C. Cruz \& J.G. Machado-Neto, 2005. Efeito da presença de sedimento na toxicidade aguda do sulfato de cobre e do triclorfon para três espécies de Daphnia. Pesticidas: Revista de Ecotoxicologia e Meio Ambiente, 15: 55-64.

Beretov, M.A. \& M. Liess, 2006. Acute contamination with esfenvalerate and food limitation: Chronic effects on the mayfly, Cloeon dipterum. Environmental Toxicology and Chemistry, 24: 1281-1286.

Beretov, A. \& F. Ribeyre, 1989. Fundamentals concepts in aquatic ecotoxicology. In: A. Boudou; Ribeyre, F. (eds). Aquatic ecotoxicology, fundamentals concepts and methodologies, 1: 35-75.

Couceiro, S. R. M., B.R. Forsberg, N. Hamada \& R.L. Ferreira, 2006. Os efeitos de um vazamento de óleo e despejo de esgoto doméstico na fauna de insetos do igarapé Cururu, em Manaus, Amazonas, Brasil. Brazillian. Journal Biology, 6635-44.

Eysink, G.G.J., H.B. De Pádua, S.A.E. Piva-Bertoletti, M.C. Martins, D.N. Pereira \& S. Roberto, 1988. Metais pesados no vale do Ribeira e Iguapé, Cananéia. Revista Ambiente, 2: 613.

INPESC, 2007. Otoxicologia. Disponível em: http://www.inpesc.cl.ecotoxicologia.html. Acesso: 28/01/2007.

Mccaffeerty, W. P., 1981. Aquatic Entomology . The Fishermens and Ecologists Illustrated guide to Insects and their Relatives. Jones and Bartlett Publishers, Inc. Portolla Valley, Boston. 448p.

Melo, E.G.F., M.S.R. Silva \& S.A.F. Miranda, 2005. Influência Antrópica Sobre Águas de Igarapés na Cidade De Manaus - Amazonas. Caminhos de Geografia, 5: 40-47

Michaud, A.L., L. Hare \& P.G.C. Campbell, 2005. Exchange rates of cadmium between a burrowing mayfly and its surroundings in nature. Limnology and Oceanography, 50: 1707-1717.

Miesbauer, H., G. Köck \& L. Füreder, 2001. Determination of trace elemente in macrozoobenthos samples by totalreflection X-ray fluorescence analysis. Spectrochimica Acta Part B, 56:2203 -2207.

Sampaio, A. Q. \& G.P. Santana, 2000. Metais pesados $(\mathrm{Cu}, \mathrm{Cd}$, $\mathrm{Zn}, \mathrm{Mn}, \mathrm{Fe}, \mathrm{Pb}$ e $\mathrm{Cr}$ ) em água na região do Distrito Industrial de Manaus (AM). In: XL Congresso Brasileiro de Química, Recife.

Silva, M.S.R., 1999. Metais de transição nos sedimentos de igarapés de Manaus-AM. Acta Limnológica Brasiliensia, 11: 89-100.

Siqueira, G.W. \& E.S. Braga, 2000. Avaliação da dinâmica e da biodisponibilidade de $\mathrm{Zn}, \mathrm{Ni}$, Co e $\mathrm{Pb}$ para a biota a partir de sedimentos da plataforma continental do Amapá, Nordeste da Amazônia. In: E.V.G. Espíndola, C.M.R.B. Paschoal, O. Rocha, M.B.C. Bohrer \& A.L. Oliveira-Neto. (eds). Ecotoxicologia. Perspectivas para o século XXI. RiMA editora, São Carlos, São Paulo, Brasil. 241-265.

Tonissi, F.B. \& E.L.G Espíndola, 200o. Utilização de bioensaios agudo, crônico-parcial e In situ com Danio rerio para avaliação ecotoxicológica do reservatório de Salto Grande (Americana, SP). In: E.V.G. Espíndola, C.M.R.B. Paschoal, O. Rocha, M.B.C. Bohrer \& A.L. Oliveira-Neto (eds). Ecotoxicologia. Perspectivas para o século XXI. RiMA editora, São Carlos, São Paulo, Brasil. 483-498.

Recebido em: 22/01/2008

Aceito em: 04/06/2008 $* * * * * * * * * * * * *$ 


\section{Como citar este artigo:}

Belmont, E.L.L., M.J.N.Lopes, M.S.R. Silva \& C.R. Santos-Neto 2008.

Bioensaio com náiades de Campylocia anceps Eaton, 1983 para

avaliação dos metais cobre (Cu) e zinco (Zn). EntomoBrasilis, 1(2): 33-35.

www.periodico.ebras.bio.br/ojs

(c) 890

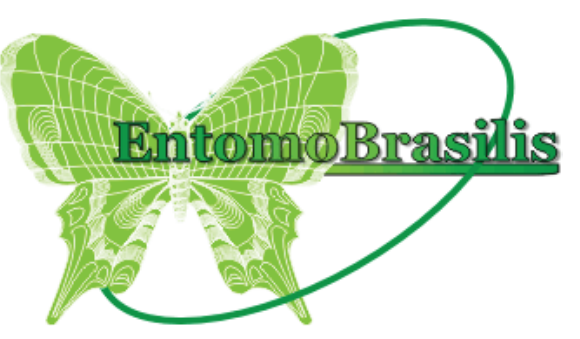

